\title{
Adolescentes y jóvenes portadores de cardiopatías congénitas en etapa de transferencia a la atención médica de adultos
}

\author{
Adolescents and young adults with congenital heart defects in transfer to adult care
}

\author{
Macarena Soto V. ${ }^{\text {a }}$, Tamara Zubarew G..$^{\text {, Ma. Francisca Arancibia-Galilea }}{ }^{\mathrm{c} d}$ \\ aDivisión de Pediatría, Facultad de Medicina, Pontificia Universidad Católica de Chile. Santiago, Chile \\ bUnidad de Adolescencia, División de Pediatría, Facultad de Medicina, Pontificia Universidad Católica de Chile. Santiago, Chile \\ ‘Departamento de Cardiología Pediátrica y Enfermedades Respiratorias del Niño, División de Pediatría, Facultad de Medicina, \\ Pontificia Universidad Católica de Chile. Santiago, Chile \\ ¿Unidad de Cardiopatías Congénitas del Adulto, Instituto Nacional del Tórax. Santiago, Chile
}

Recibido: 24 de julio de 2019; Aceptado: 13 de febrero de 2020

¿Qué se sabe del tema que trata este estudio?

Adolescentes con enfermedades crónicas tienen mayor riesgo de complicaciones potencialmente graves de su enfermedad por falta de adherencia y continuidad de sus controles. El período más crítico es el de transferencia del cuidado a los servicios de adultos.

\section{¿Qué aporta este estudio a lo ya conocido?}

Es el primer estudio a nivel nacional que reporta el nivel de conocimiento y manejo de enfermedad en adolescentes con cardiopatía congénita. El desconocimiento y escaso automanejo hace imperativa la implementación de programas planificados de transición al cuidado adulto en los servicios de cardiología pediátrica.

\section{Resumen}

Las cardiopatías congénitas (CC), la malformación congénita más frecuente, han experimentado un aumento de sobrevida y crecimiento exponencial de la población de adolescentes y adultos portadores de CC. Para el éxito a largo plazo urgen intervenciones que optimicen la transición de cuidados de salud desde los servicios pediátricos a los de adulto. Objetivo: Describir el conocimiento y manejo de la enfermedad, autoeficacia y calidad de vida en adolescentes y jóvenes con CC en periodo de transferencia en dos hospitales en Santiago de Chile. Pacientes y Método: Estudio no experimental, descriptivo, de corte transversal. Se aplicó: a) Encuesta de datos sociodemográficos, conocimiento y manejo de su enfermedad y uso de servicios de salud; b) Escala Con-Qol de Calidad de Vida Relacionada con Salud (CVRS) en pacientes con CC y c) Escala de Autoeficacia Generalizada (EAG). Resultados: Se obtuvo una muestra de 51 pacientes, $53 \%$ hombres, edad promedio de $17 \pm 2,49$ años. El $22 \%$ de las CC fue de complejidad simple, $29 \%$ moderada y $49 \%$ alta. Presentaron alta autoeficacia y buenos niveles de calidad de vida, sin embargo, mostraron escaso conocimiento y manejo de su enfermedad cardiaca. Conclusiones: Destaca la poca preparación para lograr una transición exitosa a servicios de adultos y jóvenes portadores de CC, siendo fundamental implementar programas de transición centrados en educación, autocuidado y automanejo de la enfermedad.

Correspondencia:

Ma. Francisca Arancibia-Galilea

mfaranci@uc.cl

Palabras clave:

Calidad de vida; autoeficacia; transición; cardiopatías congénitas; adolescentes 


\begin{abstract}
Congenital heart defects (CHD), the most frequent congenital malformations, have shown an increased survival and exponential growth of the adolescent and adult population living with CHD. Interventions that optimize the transition of patients from pediatric to adult health care services are essential for ensuring positive long-term outcomes. Objective: To describe the knowledge and management of this disease, self-efficacy, and quality of life of young people with CHD during the transition period in two hospitals in Santiago, Chile. Patients and Method: Non-experimental, descriptive, cross-sectional study. Patients completed: a) a survey of socio-demographic data, knowledge and management of their condition, and use of health services; b) the Health-Related Quality of Life (Con-HRQoL) Scale in patients with CHD; and c) the Generalized Self-Efficacy (GSE) Scale. Results: We obtained a sample of 51 patients, $53 \%$ of them were men, and the mean age was $17 \pm 2.49$ years. The complexity of the CHD was mild in $22 \%$, moderate in $29 \%$, and high in $49 \%$. Although patients reported high self-efficacy and good levels of quality of life, there was a lack of knowledge and self-management of their heart disease. Conclusions: The study showed that adolescents and young people with CHD are not prepared to achieve a successful transition to adult health care services, and there is a need for the implementation of transition programs focused on education, self-care, and self-management of the disease.
\end{abstract}

\section{Keywords:}

Quality of life; self-efficacy; transition; congenital heart defects; adolescents

\section{Introducción}

Las cardiopatías congénitas (CC) son la malformación congénita más frecuente. En Chile se estima una prevalencia de $8-10$ por cada 1000 nacidos vivos, similar a cifras internacionales ${ }^{1}$. Los grandes avances en cardiología y cardiocirugía pediátrica durante los últimos 60 años han dado como resultado un crecimiento exponencial de la población de adolescentes y adultos portadores de $\mathrm{CC}^{2}$. La sobrevida a la adultez en esta población ha aumentado desde un $25 \%$ a más del $95 \%$ en los últimos $40 \mathrm{años}^{3}$. Este nuevo perfil epidemiológico hace necesario poner el foco en el logro de una transferencia exitosa de adolescentes portadores de CC desde los servicios pediátricos a la atención médica de adultos. En nuestro país, no existen servicios de transición para adolescentes portadores de CC.

La transición de cuidados en salud es el proceso progresivo de movimiento planificado de adolescentes y adultos jóvenes con condiciones físicas crónicas, desde una atención orientada al niño a un sistema de cuidados de salud orientado al adulto, contemplando factores físicos y psicosociales ${ }^{4}$. La transferencia, en cambio, es un evento, que consiste en el movimiento de pacientes y registros médicos de un centro de seguimiento pediátrico a otro de adultos. Se ha demostrado que la pérdida de seguimiento alrededor del período de transferencia pone a los pacientes en riesgo de presentar complicaciones de su enfermedad, comorbilidades y necesidad de cuidados urgentes por descompensaciones ${ }^{5}$. En estudios internacionales realizados en población portadora de CC, se ha observado una pérdida de seguimiento de hasta un $50 \%$ en el período de transferencia, con hasta un $7 \%$ de pacientes que no recibió ningún tipo de atención médica posterior a su transferencia desde servicios pediátricos ${ }^{2}$.

Por otro lado, en adolescentes portadores de enfermedades crónicas se ha descrito que un mayor conocimiento de su condición y/o enfermedad, se asocia con mayores habilidades de desarrollo personal, como autoeficacia, lo que, a su vez, se relaciona a un mejor automanejo y mejores niveles de bienestar y calidad de vida ${ }^{6}$.

El objetivo de esta investigación es describir el conocimiento y manejo de su enfermedad, grado de autoeficacia y calidad de vida alrededor del período de transferencia a la atención médica de adultos, en adolescentes y jóvenes portadores de CC en control en dos hospitales en Santiago de Chile.

\section{Pacientes y Método}

Para este estudio se utilizó un diseño no experimental, descriptivo, de corte transversal. Al momento del estudio en ninguno de los centros estudiados existe un proceso formal y planificado de transición de servicios pediátricos a adultos.

Se incluyeron adolescentes y jóvenes de 13 a 21 años, consultantes entre julio de 2018 y mayo de 2019, con diagnóstico de CC, y que se encontraran en seguimiento en:

- Departamento de Cardiología Pediátrica de un hospital general universitario (Red de Salud UCChristus), grupo de adolescentes en período de pre transferencia. 
- Unidad de Cardiopatías Congénitas del Adulto de un hospital público de especialidad en Santiago de Chile (Instituto Nacional del Tórax), grupo de adolescentes y jóvenes en período de post-transferencia.

Se excluyeron pacientes que por sus condiciones cognitivas no pudieran dar respuesta por escrito a las encuestas requeridas.

El estudio contó con la aprobación del Comité Ético Científico de la Facultad de Medicina de la Pontificia Universidad Católica de Chile y del Comité Ético Científico del Servicio de Salud Metropolitano Oriente. Se solicitó el consentimiento informado del cuidador y el asentimiento en el caso de ser menor de edad y consentimiento informado en los mayores de edad.

Los instrumentos fueron auto aplicados en la sala de espera, previa explicación al paciente y supervisado por algún miembro del equipo de investigación en caso de existir dudas.

Se aplicaron los siguientes instrumentos:

a) Encuesta estructurada que incluyó: datos sociodemográficos generales, información respecto a su enfermedad y controles, conocimiento de su condición médica y tratamiento, conocimiento respecto al uso de servicios de salud y percepción respecto a la toma de decisiones en salud. De acuerdo con el diagnóstico clínico, se definió la complejidad de CC, según criterios de Bethesda, en simple, moderada y alta complejidad ${ }^{7}$.

b) Escala Con-Qol de Calidad de Vida Relacionada a Salud (CVRS). Fue desarrollada para niños y adolescentes portadores de CC y ha sido validada en Chile para su aplicación en adolescentes ${ }^{8}$. Incluye la medición de cuatro dominios: a) síntomas (frecuencia e intensidad); b) limitación en actividades cotidianas; c) relaciones y d) enfrentamiento y control de la enfermedad. Arroja puntajes de 0 a 100 , donde 0 indica la peor CVRS y 100 la mejor CVRS.

c) Escala de Autoeficacia Generalizada (EAG). Mide la percepción personal respecto de las capacidades propias para manejar en su vida diferentes situaciones estresantes ${ }^{9}$ y fue validada en Chile para su aplicación en población general mayor de 15 años ${ }^{10}$. La escala consta de 10 preguntas, con puntaje mínimo de 10 y máximo de 40 puntos. Ejemplo de preguntas son: "Siempre puedo resolver problemas difíciles si me esfuerzo lo suficiente"; "Es fácil para mí cumplir mis objetivos”. Las posibles respuestas son: $1=$ incorrecto, $2=$ apenas cierto, $3=$ más bien cierto y $4=$ cierto. A mayor puntaje, mayor autoeficacia general percibida. Los autores de la escala refieren que no hay un punto de corte preestablecido y sugieren dicotomizar la muestra en 30 puntos si la mediana de la muestra es cercana a ese valor, o establecer grupos sobre la base de las distribuciones empíricas de una población de referencia en particular. Los valores obtenidos en la validación chilena de la EAG fueron promedio 34,18 puntos \pm 4.84 y mediana 35 puntos (rango $16-40)^{10}$.

\section{Análisis estadístico}

Los datos obtenidos fueron manejados de manera anonimizada por el investigador responsable, quién se encargó de la digitalización y análisis, apoyado por los co-investigadores. En ningún caso se almacenaron datos que permitieran la identificación de los sujetos de investigación.

Para estadística descriptiva se utilizó número y porcentaje para variables categóricas o nominales y promedio y desviación estándar para variables continuas. Se aplicó la prueba $\chi^{2}$ de Pearson para variables categóricas y comparación de promedios de t de Student para variables continuas. Se consideró estadísticamente significativo un valor $\mathrm{p}<0,05$.

Para evaluar asociación se utilizó análisis de regresión lineal múltiple.

Todos los análisis fueron realizados mediante el software IBM SPSS Statistics ${ }^{\circledR}$ (Chicago, IL, USA), versión 26.0 .

\section{Resultados}

Se obtuvo una muestra de 51 adolescentes y jóvenes portadores de CC: 15 adolescentes en período pre-transferencia en seguimiento en el Departamento de Cardiología Pediátrica en un hospital general universitario y 36 adolescentes y jóvenes en período posttransferencia en control en la Unidad de Cardiopatías Congénitas del Adulto de un hospital público de especialidad.

Del total de pacientes, 53\% correspondió al sexo masculino. La edad promedio fue $17 \pm 2,49$ años, siendo de $14 \pm 1,41$ años en el grupo de pacientes evaluados en el Departamento de Cardiología Pediátrica y de $18 \pm 1,80$ años en la Unidad de Cardiopatías Congénitas del Adulto.

Un 53\% del total se reconoció como beneficiario del sistema público de previsión de salud, 12\% del sistema privado de previsión de salud y el 35\% de los pacientes desconocía su sistema de previsión.

Del total de adolescentes y adultos jóvenes, un 49\% $(\mathrm{n}=25)$ de los pacientes presentaba CC de alta complejidad, 29\% $(\mathrm{n}=15)$ de complejidad moderada y $22 \%(n=11)$ simple. El $82 \%$ de los pacientes habían sido sometidos a algún tipo de cirugía cardiaca en la edad pediátrica. 
En cuanto a nivel educacional, aproximadamente la mitad de la muestra cursaba educación media científico humanista. Las características sociodemográficas de la población se describen en la tabla 1 .

Con respecto a la evaluación de conocimiento de su enfermedad, la mayoría declaró saber el nombre de su enfermedad y medicamentos prescritos. Sin embargo, un $83 \%$ declaró no conocer o conocer parcialmente las reacciones adversas de sus fármacos. Entre 30 y 40\% de los entrevistados declaró no conocer a cabalidad el efecto del consumo de tabaco, drogas y alcohol en su enfermedad cardíaca. Destacó que alrededor de un tercio de las pacientes de sexo femenino, declaró no saber si era seguro cursar un embarazo considerando su condición cardiovascular (tabla 2).

Con respecto al manejo de su enfermedad, el $84 \%$ de los pacientes refirió participar en las decisiones relacionadas con su salud. Sin embargo, casi la mitad de la muestra refirió recibir los medicamentos por parte del cuidador, un 50\% reconoció no saber cómo ubicar a su médico y un 57\% manifestó desconocimiento con respecto a su previsión de salud a futuro (tabla 3 ).

$\mathrm{Al}$ analizar por edad, no se encontró diferencias significativas en la mayoría de las preguntas de conocimiento, manejo de enfermedad y utilización de servicios de salud, entre el grupo de adolescentes de 17 años y menos y el grupo de mayores de 17 años. Sólo se observó que el grupo de mayor edad declaró mayor conocimiento respecto al efecto de los medicamentos utilizados $(\mathrm{p}=0,009)$ y una mayor percepción de su participación en la toma de decisiones en salud $(\mathrm{p}=0,021)$. Al comparar el grupo de pacientes en período de pre-transferencia versus post-transferencia, tampoco se observaron diferencias en el conocimiento, manejo de enfermedad y uso de servicios de salud.

La puntuación media para la EAG fue de 31,3 \pm

Tabla 1. Descripción sociodemográfica de adolescentes y adultos jóvenes portadores de CC

\begin{tabular}{|c|c|c|c|c|}
\hline & $\begin{array}{c}\text { Total } \\
n=51 \\
n(\%)\end{array}$ & $\begin{array}{l}\text { Departamento Cardiología Pediátrica } \\
\qquad \begin{array}{c}\mathrm{n}=15 \\
(\%)\end{array}\end{array}$ & $\begin{array}{l}\text { Unidad CC del adulto } \\
\qquad \begin{array}{c}n=36 \\
n(\%)\end{array}\end{array}$ & Valor $\mathrm{p}$ \\
\hline \multicolumn{5}{|l|}{ Género } \\
\hline Femenino & $24(47)$ & $7(47)$ & $17(47)$ & NS \\
\hline Masculino & $27(53)$ & $8(53)$ & $19(53)$ & NS \\
\hline Edad (años) & $17 \pm 2,49$ & $14 \pm 1,41$ & $18 \pm 1,8$ & $<0,0001$ \\
\hline \multicolumn{5}{|l|}{ Complejidad CC* } \\
\hline Simple & $11(22)$ & $2(13)$ & $9(25)$ & NS \\
\hline Moderada & $15(29)$ & $3(20)$ & $12(33)$ & NS \\
\hline Alta & $25(49)$ & $10(67)$ & $15(42)$ & NS \\
\hline \multicolumn{5}{|l|}{ Nivel educacional actual } \\
\hline Escuela especial o diferencial & $3(6)$ & 0 & $3(8)$ & NS \\
\hline Básica o primaria & $6(12)$ & $6(40)$ & 0 & 0,0001 \\
\hline Ed. media científico humanista & $23(45)$ & $8(53)$ & $15(42)$ & NS \\
\hline Ed. media técnico profesional & $3(6)$ & 0 & $3(8)$ & NS \\
\hline Ed. Universitaria & $7(14)$ & $1(7)$ & $6(17)$ & NS \\
\hline Ed. Técnica superior & $9(17)$ & 0 & $9(25)$ & NS \\
\hline \multicolumn{5}{|l|}{ Previsión } \\
\hline Sistema Público & $27(53)$ & $5(33)$ & $22(61)$ & NS \\
\hline Sistema Privado & $5(10)$ & $5(33)$ & 0 & 0,0005 \\
\hline Otros & $1(2)$ & 0 & 1 (3) & NS \\
\hline No sé & $18(35)$ & $5(33)$ & $13(36)$ & NS \\
\hline \multicolumn{5}{|l|}{ Estado civil } \\
\hline Casado & $1 \quad(2)$ & 1 (7) & 0 & NS \\
\hline Conviviente & 1 (2) & 0 & 1 (3) & NS \\
\hline Soltero & $49(96)$ & $14(93)$ & $35(97)$ & NS \\
\hline \multicolumn{5}{|l|}{ Región } \\
\hline Metropolitana & $7(14)$ & $4(27)$ & $3(8)$ & NS \\
\hline Otras & $40(86)$ & $11(73)$ & 33 (92) & NS \\
\hline
\end{tabular}

Se consideró estadísticamente significativo $p<0,05 .{ }^{*}$ Complejidad de cardiopatías congénita según criterios Bethesda ${ }^{7}$. C $=$ Cardiopatías congénitas; NS = No significativo; Ed. = Educación. 
Tabla 2. Grado de conocimiento de su enfermedad en adolescentes y adultos jóvenes portadores de CC

\begin{tabular}{|c|c|}
\hline & $\begin{array}{c}\text { Total } \\
(n=51) \\
n(\%)\end{array}$ \\
\hline \multicolumn{2}{|c|}{ ¿Conoces el nombre de tu enfermedad cardíaca? } \\
\hline Sí & $40(78)$ \\
\hline No & $11(22)$ \\
\hline \multicolumn{2}{|c|}{ ¿Conoces el nombre de tus medicamentos? } \\
\hline Sí & $25(86)$ \\
\hline Parcialmente & $4(14)$ \\
\hline \multicolumn{2}{|c|}{$\begin{array}{l}\text { ¿Conoces para qué sirven los medicamentos que } \\
\text { usas? }\end{array}$} \\
\hline Sí & $20(69)$ \\
\hline No & $3(10)$ \\
\hline Parcialmente & $6(21)$ \\
\hline \multicolumn{2}{|c|}{ ¿Conoces las reacciones adversas de tus fármacos? } \\
\hline Sí & $5(17)$ \\
\hline No & $20(69)$ \\
\hline Parcialmente & $4(14)$ \\
\hline \multicolumn{2}{|l|}{ ¿Sabes si es seguro embarazarte? } \\
\hline De acuerdo & $14(59)$ \\
\hline Moderadamente de acuerdo & $2(8)$ \\
\hline En desacuerdo & $8(33)$ \\
\hline \multicolumn{2}{|c|}{$\begin{array}{l}\text { ¿Conoces el efecto del tabaco en tu enfermedad } \\
\text { cardíaca? }\end{array}$} \\
\hline De acuerdo & $33(65)$ \\
\hline Moderadamente de acuerdo & $12(23)$ \\
\hline En desacuerdo & $6(12)$ \\
\hline \multicolumn{2}{|c|}{$\begin{array}{l}\text { ¿Conoces el efecto de las drogas en tu enfermedad } \\
\text { cardíaca? }\end{array}$} \\
\hline De acuerdo & $34(67)$ \\
\hline Moderadamente de acuerdo & $8(15)$ \\
\hline En desacuerdo & $9(18)$ \\
\hline \multicolumn{2}{|c|}{$\begin{array}{l}\text { ¿Conoces el efecto del alcohol en tu enfermedad } \\
\text { cardíaca? }\end{array}$} \\
\hline De acuerdo & $31(61)$ \\
\hline Moderadamente de acuerdo & $7(14)$ \\
\hline En desacuerdo & $13(25)$ \\
\hline
\end{tabular}

Tabla 3. Manejo de la enfermedad en adolescentes y adultos jóvenes portadores de CC

\begin{tabular}{lc}
\hline & $\begin{array}{c}\text { Total } \\
(\mathrm{n}=51) \\
\mathrm{n}(\%)\end{array}$ \\
\hline $\begin{array}{l}\text { ¿Participas en la toma de decisiones en relación con } \\
\text { tu salud? }\end{array}$ & $43(84)$ \\
Sí & $8(16)$ \\
No & \\
¿Quién administra los medicamentos? & $16(55)$ \\
Yo & $13(45)$ \\
Cuidador /a & \\
¿Sabes a qué centro médico debes acudir en caso & \\
de emergencia? & $33(65)$ \\
Sí & $18(35)$ \\
Necesito aprender más & \\
¿Sabes cómo ubicar a tu médico? & $25(49)$ \\
Sí & $26(51)$ \\
No & \\
¿Sabes si debes conservar tu plan de salud de por & \\
vida? & \\
Sí & $29(57)$ \\
No & $22(43)$ \\
\hline CC $=$ Cardiopatías congénitas & \\
&
\end{tabular}

4,1 puntos, comparable a lo reportado en población general chilena. La puntuación media para Con-Qol CVRS fue de $72,5 \pm 10,7$, lo que es similar al subgrupo con mejor calidad de vida de la muestra de pacientes utilizada en la validación nacional del instrumento.

El análisis de correlación de Pearson mostró significancia bilateral entre los puntajes obtenidos en la EAG y ConQol CVSR $(p=0,012)$. Es decir, a mayor autoeficacia de los individuos, mayor es su calidad de vida declarada, con una fuerza de asociación moderada $(r=0,342)$.

El análisis de regresión múltiple mostró que la edad fue el único factor predictor significativo de autoeficacia $(\mathrm{p}=0,0420)$ con un $\mathrm{r}^{2}$ ajustado de 0,021 (tabla 4).

Tabla 4. Análisis de correlación regresión múltiple entre variables específicas y puntaje de Escala autoeficacia generalizado

\begin{tabular}{|c|c|c|c|}
\hline Variables & B & SE B & $\mathrm{p}$ \\
\hline Complejidad CC & $-0,281$ & 0,682 & 0,682 \\
\hline Edad & $-0,542$ & 0,259 & 0,042 \\
\hline Nivel de estudios & 0,489 & 0,296 & 0,105 \\
\hline Sexo & $-0,574$ & 1,152 & 0,621 \\
\hline$R^{2}$ & \multicolumn{2}{|c|}{0,021} & - \\
\hline$F$ & \multicolumn{2}{|c|}{1,288} & 0,28 \\
\hline
\end{tabular}

Se consideró estadísticamente significativo $\mathrm{p}<0,05$. CC = cardiopatías congénitas; $\mathrm{B}=$ Coeficiente; SE $\mathrm{B}=$ Error estándar del coeficiente; $\mathrm{R}^{2}=$ Coeficiente de determinación ajustado; $\mathrm{F}$ = Índice $\mathrm{F}$. 


\section{Discusión}

En base a los resultados encontrados se evidencia un escaso conocimiento de los pacientes con CC en relación con su enfermedad y farmacoterapia, tanto en adolescentes en período de pre-transferencia como adolescentes y jóvenes en post-transferencia. Esto puede ser explicado por la ausencia de un proceso planificado de transición de adolescentes desde la atención pediátrica a adultos en los centros estudiados.

El hecho de que aproximadamente un tercio de la muestra no conoce o conoce parcialmente el efecto de los fármacos que utiliza, podría representar un factor de riesgo de pérdida de adherencia al tratamiento, con las implicancias que esto tiene en la evolución clínica. Aún más alarmante es el nivel de desconocimiento de las reacciones adversas de sus fármacos, pudiendo ser potencialmente deletéreo en su salud. El desconocimiento de las mujeres de la muestra con respecto a la seguridad de cursar un embarazo con su condición cardiovascular es alto. Las contraindicaciones de embarazo en la población de mujeres con cardiopatías congénitas se encuentran limitadas a un grupo pequeño de casos, sin embargo, la recomendación es que los embarazos sean planificados y con chequeo previo de su condición cardiovascular ${ }^{11}$. El desconocimiento de las pacientes en esta área las expone a riesgos innecesarios en el caso de embarazos no planificados en población de riesgo y también en el caso de mujeres que, sin tener una contraindicación para embarazo o parto normal, se enfrentan a la situación de un embarazo que puede ser altamente estresante por el miedo percibido por su condición cardiovascular, con eventuales intervenciones obstétricas innecesarias y riesgosas (reposo, inducciones pretérmino, cesárea electiva), que en la mayoría de las pacientes no se justifican clínicamente por su enfermedad cardíaca.

Existe un pobre automanejo de la enfermedad actual, ejemplificado en que solo la mitad de la muestra total se administra personalmente sus fármacos, donde se evidencia la presencia permanente de una díada cuidador-paciente que ha extendido su modelo de funcionamiento pediátrico, sin existir un cambio en la asignación de responsabilidades de cuidado de acuerdo con la maduración del adolescente. Además, hay un escaso grado de conocimiento del funcionamiento clínico y administrativo de los servicios de salud, destacando que la mayoría de los pacientes declara no saber cómo contactar a su médico ni solicitar hora de control. Un 35\% de los adolescentes y jóvenes portadores de CC señala no saber dónde acudir en caso de emergencia, lo que podría representar una demora en el caso de requerir este tipo de atención.

La falta de conocimiento pudiese explicarse por un modelo de atención pediátrico paternalista, donde tanto padres como personal de salud entregan escasa información a los adolescentes y jóvenes, siendo el cuidador quien asume el rol principal en el manejo de la enfermedad hasta muy avanzada edad del paciente ${ }^{12}$.

Como un aspecto positivo, destaca la alta percepción de participación de adolescentes y jóvenes portadores de CC en la toma de decisiones con relación a su salud. La autoeficacia general percibida por los pacientes es similar a lo reportado en población sana ${ }^{10}$, mostrando que la población estudiada presenta un nivel aceptable en este dominio con relación al manejo de situaciones generales de vida, lo que no tiene necesariamente relación al manejo de su condición de salud. En estudios internacionales se ha demostrado que la presencia de alta autoeficacia en adolescentes portadores de CC se relaciona con una transferencia exitosa a servicios de salud de adultos ${ }^{6}$.

El buen nivel de autoeficacia en este estudio podría explicarse por varias razones: 1) características de omnipotencia e invulnerabilidad, propias de la etapa adolescente; 2) una probable separación en la percepción de estos pacientes entre su vida diaria y su condición de salud, es decir, se perciben autoeficaces en aspectos cotidianos de su vida, sin considerar en las respuestas aspectos relativos a su condición de salud o 3) la presencia permanente de una figura parental sobreprotectora que resuelve todas sus limitaciones y dificultades. Sin embargo, estas conjeturas no se pueden derivar de este estudio y se requiere de otro tipo de metodología para ello.

Con respecto a Con-QoL CVRS, el puntaje obtenido por nuestra muestra es comparable al puntaje obtenido en los pacientes que reportaron una autopercepción de salud "Muy buena" o "Buena" en la población de validación chilena, mostrando que nuestra muestra declara tener niveles de calidad de vida relacionada a su salud dentro del rango alto, comparado con población adolescente portadora de CC de referencia nacional ${ }^{8}$.

El estudio muestra que, a mayor autoeficacia de los individuos, mayor es su calidad de vida declarada, lo que es consistente con lo publicado en estudios internacionales ${ }^{6}$. También se observa que la autoeficacia se correlaciona significativamente con la edad del paciente, existiendo mayor autoeficacia en pacientes mayores. Sin embargo, el coeficiente de determinación ajustado para autoeficacia fue bajo, mostrando que podrían existir otras variables que expliquen la autoeficacia y que no hayan sido incluidas en el modelo.

El hallazgo de un adecuado nivel de autoeficacia permite asumir que los adolescentes y jóvenes portadores de CC tienen un alto potencial de automanejo de su enfermedad ${ }^{10}$. Sin embargo, muestran escaso conocimiento de su enfermedad y de la utilización de los servicios de salud. Estos hallazgos son relevantes para las políticas públicas en nuestro país ya que realzan la 
necesidad de implementar programas planificados de transición de adolescentes portadores de CC desde los servicios pediátricos a adultos, enfocados en la entrega de conocimiento de su enfermedad y manejo, en la prevención de conductas de riesgo y en educación respecto a la utilización de servicios de salud.

Se debe entender que la transición es un proceso planificado, que debe iniciarse en la edad pediátrica y extenderse hasta después del momento de la transferencia, terminando cuando el paciente tenga desarrolladas las competencias necesarias para ser autónomo en relación con su condición de salud, con todo lo que esto implica.

En cuanto a las limitantes del estudio, la muestra incluye sólo a dos hospitales de la Región Metropolitana de Santiago, por lo que los resultados no son extrapolables a la población total de adolescentes y jóvenes portadores de CC. Además, se trata de una muestra seleccionada de pacientes adherentes a controles de salud, por lo que se podría presumir que la población no adherente a control tiene aún peor conocimiento de su condición de salud y manejo de su enfermedad, llegando al extremo de la pérdida de seguimiento. Es también una limitante el tamaño muestral, sobre todo de pacientes en período de pre-transferencia, lo que limita la significancia del análisis estadístico.

A pesar de estas limitaciones, los resultados de este estudio contribuyen a nuestra comprensión de la situación actual de los adolescentes y jóvenes portadores de CC en el período de transferencia. Son necesarios futuros estudios multicéntricos representativos de las diferentes regiones del país para obtener resultados generalizables a toda la población de adolescentes y jóvenes portadores de CC en período de transferencia a la atención adulta.

\section{Conclusiones}

Este estudio muestra la falta de preparación para la transferencia a los cuidados de salud del adulto en esta población de adolescentes y jóvenes portadores de CC, concordante con lo que se ha descrito en los estudios actuales de adolescentes portadores de enfermedades crónicas en proceso de transición ${ }^{12}$.

Dado el cambio de perfil epidemiológico que se enfrenta actualmente con relación a patologías cróni- cas de la infancia, el proceso de transición se ha vuelto un tema de máxima relevancia a nivel nacional e internacional. Urge la necesidad de implementación de programas y servicios de transición de adolescentes y jóvenes portadores de CC, centrados en autocuidado y automanejo de su enfermedad, que proporcionen apoyo a las familias, a los servicios de salud y a la comunidad, todos quienes influirán en la transición exitosa a la atención de salud del adulto ${ }^{13}$.

Esperamos contribuir al conocimiento de la situación actual de adolescentes y jóvenes en este período crítico, siendo un punto inicial y fundamento para futuras investigaciones en el tema.

Se necesitan estudios de tipo cualitativos que profundicen la percepción de los pacientes y sus cuidadores respecto a sus fortalezas y necesidades en esta etapa.

\section{Responsabilidades Éticas}

Protección de personas y animales: Los autores declaran que los procedimientos seguidos se conformaron a las normas éticas del comité de experimentación humana responsable y de acuerdo con la Asociación Médica Mundial y la Declaración de Helsinki.

Confidencialidad de los datos: Los autores declaran que han seguido los protocolos de su centro de trabajo sobre la publicación de datos de pacientes.

Derecho a la privacidad y consentimiento informado: Los autores han obtenido el consentimiento informado de los pacientes y/o sujetos referidos en el artículo. Este documento obra en poder del autor de correspondencia.

\section{Conflicto de intereses}

Los autores declaran no tener conflicto de intereses.

\section{Agradecimientos}

Agradecemos a las enfermeras y estadísticos colaboradores. 


\section{Referencias}

1. Van Der Linde D, Konings E, Slager M, et al. Birth prevalence of congenital heart disease worldwide: A systematic review and meta-analysis. J Am Coll Cardiol. 2011;58(21):2241-7.

2. Reid GJ, Irvine MJ, McCrindle BW, Sananes R, Ritvo PG, Siu SC. Prevalence and Correlates of Successful Transfer from Pediatric to Adult Health Care among a Cohort of Young Adults with Complex Congenital Heart Defects. Pediatrics. 2004;113(3):e197-205.

3. Moons P, Bovijn L, Budts W, Belmans A, Gewillig M. Temporal trends in survival to adulthood among patients born with congenital heart disease from 1970 to 1992 in Belgium. Circulation. 2010;122(22):2264-72.

4. White PH, Cooley WC; Transitions Clinical Report Authoring Group; American Academy of Pediatrics; American Academy of Family Physicians; American College of Physicians. Supporting the Health Care Transition
From Adolescence to Adulthood in the Medical Home. Pediatrics. 2018;142(5):e20182587.

5. Sable C, Foster E, Uzark K, Bjornsen K, et al. Best practices in managing transition to adulthood for adolescents with congenital heart disease: the transition process and medical and psychosocial issues: a scientific statement from the American Heart Association. Circulation. 2011;123(13):1454-85.

6. Uzark K, Smith C, Donohue J, Yu S, Afton K, Norris M, Cotts T. Assessment of Transition Readiness in Adolescents and Young Adults with Heart Disease. J Pediatr. 2015;167:1233-8.

7. Webb GD, et al. 32nd Bethesda Conference: "Care of the Adult with Congenital Heart Disease". JACC. 2001. 37(5):1161-98.

8. Toledo M, Alarcón A, Bustos L, Molina J, Felipe H, Garay F, Castillo M, Pamela O. Validación del cuestionario de calidad de vida Con-Qol, en niños chilenos portadores de cardiopatías congénitas. Rev Med Chile. 2012;140(12):1548-53.
9. Schwarzer R, Jerusalem M. Generalized self-efficacy scale. In J. Weinman, S. Wright, M Johnston, Measurement in Health Psychology; A User's Portfolio. Causal and Control Beliefs 1995;pp. 35-7. Windsor, UK: NFER-NELSON.

10. Cid P, Orellana A, Barriga O. Validación de la escala de autoeficacia general en Chile. Rev Med Chile. 2010;138:551-7.

11. Regitz-Zagrosek V, Roos-Hesselink J, Bauersachs J, et al. ESC Guidelines for the management of cardiovascular diseases during pregnancy. European Heart Journal. 2018;39:3165-241.

12. Inostroza $\mathrm{C}$, Correa ML, Besoain C, et al. El proceso de transición de servicios pediátricos a adultos: visión de adolescentes hospitalizados portadores de enfermedades crónicas. Rev Chil Pediatr. 2016;87(2):110-5.

13. Uzark K, Afton K, Yu S, Lowery R, Smith C, Norris MD. Transition Readiness in Adolescents and Young Adults with Heart Disease: Can We Improve Quality of Life? J Pediatr. 2019;212:73-8. 